\title{
Evolution on the Immunopathology of Chagas Disease
}

$$
\text { Moysés Sadigursky }
$$

Faculdade de Medicina, Universidade Federal da Bahia, Av. Reitor Miguel Calmon s/nº , 40140-001 Salvador, BA, Brasil

Key words: Chagas disease - autoimmunity in Chagas disease

The pathogenesis of Chagas disease is still controversial. The lesions that occur in the acute phase of infection are initially due to rupture of the heart fibers by the Trypanosoma cruzi. When the parasite antigens sensitize the immune system, the inflammatory process is due to a sum of the traumatic mechanism and the immune reaction against the parasite.

After the acute phase and the development of the specific immune response occur an equilibrium between the host and the parasite which can last for all the life of the host. The T. cruzi as a true parasite do not kills the host and the immune system of the host do not kills all the parasites. Despite it can be detected specific humoral and cellular immune response against the T. cruzi, this parasite evades the immune response mainly penetrating and proliferating in host cells. The equilibrium phase is called indeterminate phase when occur a clearance of parasites from bloodstream and tissues. Patients present positive serology to $T$. cruzi antigens but do not present signs and symptoms of disease.

At about 10 to $15 \%$ of infected individuals develop a chronic heart disease characterized by chronic diffuse and fibrosing myocarditis with absence or very few parasites. Different mechanisms have been proposed to explain that aggressive myocarditis similar to a rejection of an allogeneic heart transplant. It has been suggested that the lesions result from gradual destruction of the heart fibers by parasites or by a mechanism of cellular hypersensitivity directed to parasite antigens which affect also the heart tissue. Another mechanism proposed is the autoimmune mechanism.

Soares and Ribeiro dos Santos presented results of several experiments about immunopathology of cardiomyopathy in experimental Chagas disease that indicate autoimmune mechanism as an strong candidate to explain the pathogenesis of chagasic myocardiopathy. They show an elegant

Fax: +55-71-245.8562

Received 9 June 1999

Accepted 9 August 1999 experiment where mice chronically infected with T. cruzi reject syngeneic heart grafts. Hearts grafted into normal or T. cruzi immunized syngeneic recipients are not rejected. The study shows that only CD4 and not CD8 T cells from chronically infected mice were able to promote rejection. Mice chronically infected with $T$. cruzi were treated with antiCD4 and anti-CD8 monoclonal antibodies before transplantation. The in vivo depletion of CD4 cells, but not CD8 cells abrogates rejection.

In another experiment it was shown that chronically infected mice treated with anti-CD4 monoclonal antibodies presented tolerance with abolishment of the chronic carditis. The opposite occurred when animals were treated with anti-CD8 or antiThy 1 . Tolerant animals presented low mortality and negative parasitemia. I can not understand why animals that had T-CD4 cells destroyed had a negative parasitemia. The expectance was to have immunodeficience similar to Aids with a high parasitemia. The phenotype of cells present in chagasic myocarditis has been controversial as in mice as in humans. Some authors refer predominance of CD4 cells and others predominance of CD8 cells. In our experience with material of necropsy of humans who died with Chagas disease (Castro-Silva \& Sadigursky) we had in $80 \%$ of patients predominance of CD4 cells, $10 \%$ with predominance of CD8 cells and $10 \%$ with predominance of macrophages. Macrophages were seen in variable number in all the cases.

Other experiments with lines of autoreactive CD4 cells from mice chronically infected with $T$. cruzi reinforce the hypothesis of autoimmunity in chronic Chagas disease.

Correa-Oliveira et al. presented "The role of the immune response on the development of human severe clinical forms of Chagas disease". The secretion of IFN $\gamma$ can be correlated with the severe cardiac form of Chagas disease. The opposite was observed for in vitro secretion of IL10. The studies indicated that as the cardiac function worsens, IFN $\gamma$ secretion by PBMC increases with a parallel decrease on secretion of IL10. They conclude that IFN $\gamma$ secretion can be correlated with the development of severe cardiac form of the disease. I believe that the high level of IFN $\gamma$ secre- 
tion indicates that an intense process of stimulation of cellular immunity is occurring. The high level dosage of IFN $\gamma$ can be useful for the diagnostic of severe cases of chronic myocarditis. Is not the high level of IFN $\gamma$ the cause of severe myocarditis but a consequence. I agree that IFN $\gamma$ is involved in the augmentation of the cytolitic potential of lymphocytes in the cardiac inflammatory process.

Patients with megaesophagus and megacolon present different kind of infections and subnutrition. The characterization of cells of the inflammatory process in those cases to correlate to pathogenicity is very difficult.

Simões-Barbosa et al. presented a very interesting experiment. They show that sequence of minicircle of kDNA of T. cruzi can integrate into the host genome. They show that a lineage of murine macrophage infected with $T$. cruzi incorporates genetic material of the parasite kinetoplast. Subclonal lines of transfected macrophage continue synthesizing proteins proper of the parasite. This phenomenon demonstrated in vitro, I believed, has not importance in vivo since it will appear to immune system as a foreign cell and destroyed. The antibodies in the chagasic sera recognized the $T$. cruzi antigens produced by transfected macrophage and they do not consist in autoreactive antibodies to proteins proper of the host cell.

If this mechanism really occur in vivo it can explain why animals treated with specific chemotherapy continue presenting positive serology. On the other hand it is possible that some macrophages are transfected with cross-reactive antigens and so are not rejected while persist the autotolerance.

The trigger mechanisms of autoimmunity are policlonal activation, anti idiotipic reaction and cross-reaction antigens present in the T. cruzi. We have demonstrated that $T$. cruzi have cross reacting antigens which induces antibody that reacts against antigens present in the cardiac muscle. One purified antigen is an ATPase $\mathrm{Ca}++$ and $\mathrm{Mg}++$ dependent. Sera of chronic chagasic patients have high titer of antibodies and also reactive cells against this antigen (Sadigursky \& Santos-Buch). We also demonstrated that heart muscle cells have receptors to $T$. cruzi that facilitate the parasite get into the cell. The immune response directed to the protein of the parasite can induces an anti idiotipic reaction that damage the heart.

Another point necessary to be stressed is that to induces an autoimmune disease is necessary the presence continuous of the antigen. The demonstration of $T$. cruzi or $T$. cruzi antigens in the host is a necessary condition to the development of auto-immune reaction. Barbosa Jr. and Andrade, demonstrated that in Chagas disease the parasites can be found in many different organs and tissues but only in the heart occur a chronic diffuse inflammatory infiltrate. This findings corroborate to the hypothesis of autoimmunity in Chagas disease. Andrade et al. also showed those dogs in indeterminate phase of Chagas disease when treated with low doses of cyclophosphamide which destroy cells suppressor function, evolved to a chronic diffuse myocarditis, indicating also an autoimmune mechanism. 